\title{
Micropillars with a controlled number of site- controlled quantum dots $\odot$
}

Cite as: Appl. Phys. Lett. 112, 071101 (2018); https://doi.org/10.1063/1.5017692

Submitted: 29 November 2017 . Accepted: 08 January 2018 . Published Online: 12 February 2018

Arsenty Kaganskiy, Fabian Gericke, Tobias Heuser, Tobias Heindel (iD, Xavier Porte, and Stephan Reitzenstein (i)

\section{COLLECTIONS}

This paper was selected as Featured
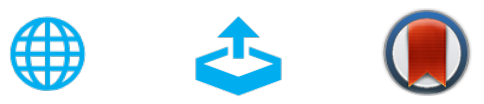

\section{ARTICLES YOU MAY BE INTERESTED IN}

Large vacuum Rabi splitting between a single quantum dot and an $\mathrm{HO}$ photonic crystal nanocavity

Applied Physics Letters 112, 093101 (2018); https://doi.org/10.1063/1.5016615

On-demand generation of background-free single photons from a solid-state source Applied Physics Letters 112, 093106 (2018); https://doi.org/10.1063/1.5020038

Guest Editorial: The dawn of gallium oxide microelectronics

Applied Physics Letters 112, 060401 (2018); https://doi.org/10.1063/1.5017845

Lock-in Amplifiers Find out more today

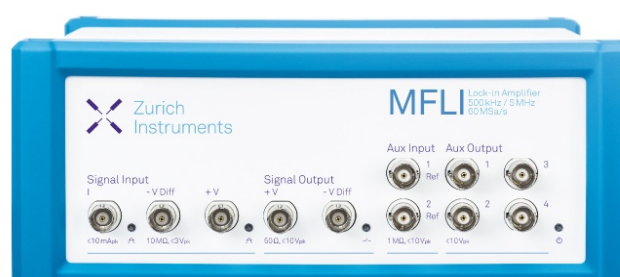

- Zurich - Instruments 


\title{
Micropillars with a controlled number of site-controlled quantum dots
}

\author{
Arsenty Kaganskiy, ${ }^{\text {a) }}$ Fabian Gericke, Tobias Heuser, Tobias Heindel, Xavier Porte, \\ and Stephan Reitzenstein \\ Institut für Festkörperphysik, Technische Universität Berlin, Hardenbergstraße 36, D-10623 Berlin, Germany
}

(Received 29 November 2017; accepted 8 January 2018; published online 12 February 2018)

\begin{abstract}
We report on the realization of micropillars with site-controlled quantum dots (SCQDs) in the active layer. The SCQDs are grown via the buried stressor approach which allows for the positioned growth and device integration of a controllable number of QDs with high optical quality. This concept is very powerful as the number and the position of SCQDs in the cavity can be simultaneously controlled by the design of the buried-stressor. The fabricated micropillars exhibit a high degree of position control for the QDs above the buried stressor and $Q$-factors of up to 12000 at an emission wavelength of around $930 \mathrm{~nm}$. We experimentally analyze and numerically model the cavity $Q$-factor, the mode volume, the Purcell factor, and the photon-extraction efficiency as a function of the aperture diameter of the buried stressor. Exploiting these SCQD micropillars, we experimentally observe a Purcell enhancement in the single-QD regime with $F_{P}=4.3 \pm 0.3$. Published by AIP Publishing. https://doi.org/10.1063/1.5017692
\end{abstract}

Enormous progress in the development of nanofabrication technologies and nanophotonic device concepts has boosted experimental and theoretical studies in the field of cavity quantum electrodynamics (cQED). ${ }^{1,2}$ A variety of devices exploiting cQED effects have been developed in recent years and include highly efficient single-photon sources (SPSs) $)^{3-8}$ as well as microlasers. ${ }^{9-12}$ Such systems are often based on semiconductor quantum dots (QDs) which are integrated into microcavities to use cQED effects for enhanced device functionality. ${ }^{13}$ Here, the huge interest in semiconductor QDs is explained by their excellent optical properties combined with the scalability of the solid-state host system. Noteworthily, the spectral and spatial matching of the emitters to the cavity mode needs to be ensured in order to maximize not only the achievable Purcell factor $F_{\mathrm{P}}{ }^{1}$ but also the related spontaneous emission factor $\beta=F_{\mathrm{P}} /$ $\left(F_{\mathrm{P}}+1\right)$, both of which are crucial parameters for cavityenhanced quantum-light sources and lasers. However, the usually applied self-organized growth mode results in a random spatial and spectral position of the QDs, which hinders a controlled fabrication of the aforementioned quantum devices. Different strategies have been developed to overcome this issue via the deterministic integration of QDs into resonators. These strategies can be divided into two groups: (a) concepts based on postgrowth alignment of resonators to selected self-assembled-grown QDs via in situ lithography approaches ${ }^{14}$ and (b) concepts based on the site-controlled growth of QDs. ${ }^{15}$ Although the first approach has been very effectively applied in recent years, it is limited to the realization of single-QD devices such as SPSs. The site-controlled approach is technologically more demanding. On the one hand, it allows for true scalability by the fabrication of ordered arrays of emitters. ${ }^{16}$ On the other hand, sitecontrolled growth methods based on etched nucleation centers typically lead to a reduced optical quality of the QDs because of enhanced non-radiative recombination and

a)arsenty.kaganskiy@tu-berlin.de spectral diffusion caused by interface states at etched surfaces. ${ }^{17}$ In addition, in this growth concept based on nano-hole arrays, the pitch between site-controlled QDs (SCQDs) is limited to about $200 \mathrm{~nm} .{ }^{18}$ In contrast, high- $\beta$ microlasers would strongly benefit from the integration of small QDensembles consisting of a defined number of emitters located at the anti-node of the laser mode. In this regard, the buriedstressor growth approach based on the partial oxidation of an AlAs aperture is highly interesting. It leads to the positioned growth of QDs aligned to a buried oxide-aperture where the number of QDs can be controlled by the aperture diameter. ${ }^{19}$ In the limit of small aperture diameters, it has already been applied for the fabrication of high-quality optically and electrically driven SPSs. ${ }^{20-22}$

In this work, we apply the buried-stressor growth concept with oxidized apertures to realize high-quality micropillar structures with a controllable number of SCQDs as gain medium. We fabricate micropillars with about 1 to 20 SCQDs positioned close to the maximum of the fundamental cavity mode. This specific configuration of the gain-medium is not feasible with any other growth and processing scheme reported to date, and is particularly attractive for the fabrication of SPSs and microlasers operating in the few-QD regime. Moreover, the presented micropillars are well suited for the study of light-matter interaction in the single-emitter regime of CQED. The present work focuses on the development of the required growth and processing technology and a detailed optical investigation of the realized SCQD micropillar cavities. It includes a systematic study of the micropillars in terms of the number of SCQDs, emission energy, effective mode volume, and quality factor $Q$ as a function of the diameter of the oxide-aperture. Using our type of micropillar, we are able to observe the Purcell enhancement for a single SCQD.

The work flow for the fabrication of micropillars comprises a two-step metal-organic chemical vapor deposition (MOCVD) epitaxial growth and cleanroom processing as schematically shown in Figs. 1(a)-1(d). First, a so-called 
(a)

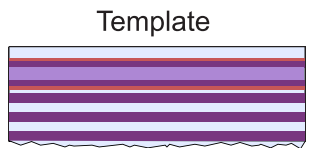

(c)

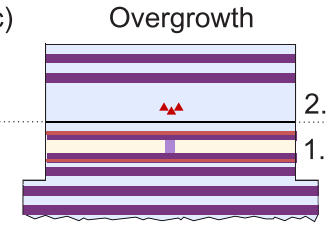

(e)

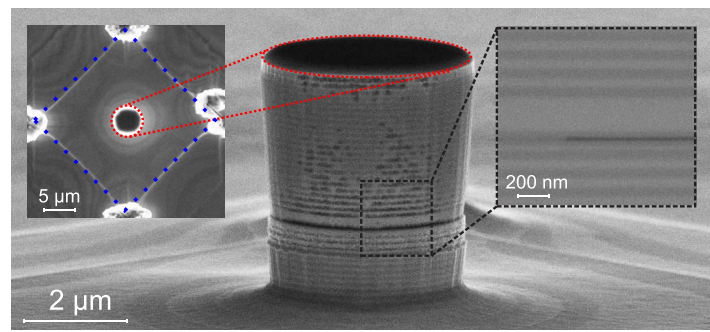

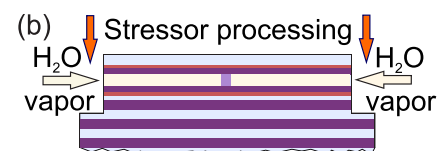

(d)

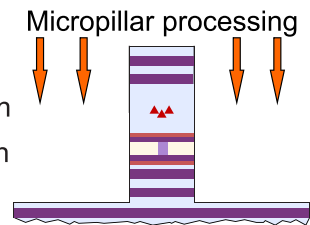

FIG. 1. (a)-(d) Schematic illustration of SCQD growth and structuring of micropillars via the buried-stressor approach. (a) The growth of a template structure is followed by mesa processing and the subsequent oxidation of the AlAs layer acting as a buried stressor (b). (c) SCQDs located in the center of a $\lambda$-cavity and the top DBR are grown in the second MOCVD step. (d) The fabrication is finalized by micropillar processing via EBL and ICP-RIE dry etching. (e) SEM image of a fully processed micropillar structure. Insets: (left) top-view of the square mesa (marked with a blue dashed square) processed in the step (b) with a micropillar (marked with a red dashed circle) aligned to its center and (right) cross-sectional SEM image of the central cavity region.

template structure is grown at $700^{\circ} \mathrm{C}$ on an n-doped GaAs substrate. It includes a lower distributed Bragg reflector (DBR) and a layer sequence consisting of a $30 \mathrm{~nm}$-thick AlAs layer embedded into $40 \mathrm{~nm}$-thick $\mathrm{Al}_{0.90} \mathrm{Ga}_{0.10} \mathrm{As}$ claddings and $10 \mathrm{~nm}$ thick strain reducing AlGaAs layers [Fig. 1(a)]. The first growth is completed by an 80 nm-thick GaAs layer representing the lower part of the $\lambda$-cavity. The DBR mirror consists of 27 pairs of $\lambda / 4$-thick $\mathrm{Al}_{0.90} \mathrm{Ga}_{0.10} \mathrm{As} / \mathrm{GaAs}-$ layers in which the thickness of the last GaAs layer is reduced to $22 \mathrm{~nm}$ in order to align the AlAs layer to an antinode of the electrical field. Next, arrays of square mesas with the side length varying between 20.4 and $21.4 \mu \mathrm{m}$ (step-size $67 \mathrm{~nm}$ ) are patterned via optical lithography and dry etching. The etching is stopped at the uppermost mirror-pair of the lower DBR in order to access the AlAs layer for the lateral oxidation [cf. Fig. 1(b)] which is subsequently performed under a $\mathrm{H}_{2} \mathrm{O} / \mathrm{N}_{2}$ atmosphere at $420^{\circ} \mathrm{C}$. Control of the aperture diameter with an accuracy of a few hundred nanometers is achieved by in situ optical monitoring during the oxidation process. Prior to the second epitaxial growth step, the sample is dipped into $75 \%$ sulfuric acid in order to remove oxides from the surface. This cleaning step is crucial for the realization of a defect-free overgrowth with the $\approx 3.5 \mu \mathrm{m}$ thick upper DBR. The MOCVD overgrowth starts with a $50 \mathrm{~nm}$ thick GaAs buffer layer grown at $700^{\circ} \mathrm{C}$ followed by $\mathrm{In}_{0.60} \mathrm{Ga}_{0.40} \mathrm{As} \mathrm{QDs}$ grown in the Stranski-Krastanov growth mode at $500{ }^{\circ} \mathrm{C}$. The growth is finalized by the upper half of the GaAs cavity with a thickness of $130 \mathrm{~nm}$ and 23 pairs of $\mathrm{Al}_{0.90} \mathrm{Ga}_{0.10} \mathrm{As} / \mathrm{GaAs}$ resembling the upper $\mathrm{DBR}$ grown at $615^{\circ} \mathrm{C}$ [cf. Fig. 1(c)]. After the overgrowth, a $300 \mathrm{~nm}$-thick $\mathrm{Si}_{3} \mathrm{~N}_{4}$ layer is deposited on the sample by plasma-enhanced

chemical vapor deposition (PECVD) followed by spin-coating of a $400 \mathrm{~nm}$ thick layer of the negative-tone resist AZ nLOF 2070. Then, micropillar structures are defined via electron beam lithography (EBL) and transferred by inductively coupled-plasma reactive-ion etching (ICP-RIE) [Fig. 1(d)]. Here, the micropillars are aligned to the center of the square mesas (corresponding to the position of the oxide-apertures) and the associated SCQDs with a high position accuracy of about $200 \mathrm{~nm}$ [cf. left inset of Fig. 1(e)]. Figure 1(e) shows a scanning electron microscopy (SEM) image of a fully processed micropillar with a diameter of $4.4 \mu \mathrm{m}$. The microcavity structure is etched down to the 17th pair of the bottom DBR, and the realized micropillar has almost vertical sidewalls with low surface roughness. The right inset of Fig. 1(e) presents a cross-sectional zoom-in view of the central part of the structure before the last dry etching step including the partially oxidized AlAs (transition from the grey AlAs to the black oxidized layer). It also shows high-quality defect-free epitaxial layers of the cavity structure.

The fabricated micropillar structures are investigated via micro-photoluminescence ( $\mu \mathrm{PL})$ spectroscopy at $10-50 \mathrm{~K}$ under continuous-wave excitation at $671 \mathrm{~nm}$. The resulting photoluminescence is collected via a microscope objective with a numerical aperture of 0.4 and detected using a spectrometer with a spectral resolution of $25 \mu \mathrm{eV}$.

In order to demonstrate and investigate the site-control of our QD-growth technique, we prepared a reference sample nominally identical to the sample described above, but where the upper DBR is replaced by a $3 \mathrm{~nm}$-thick GaAs capping layer above the SCQDs for atomic-force microscopy characterization. The results are presented in the left part of Fig. 2. As expected, ${ }^{19}$ the number of QDs increases with increasing aperture diameter, starting from 2 QDs for an aperture diameter of $700 \mathrm{~nm}$ (topmost image), leading to 9 QDs for an aperture of $1000 \mathrm{~nm}$, and resulting in a small QD-ensemble for an
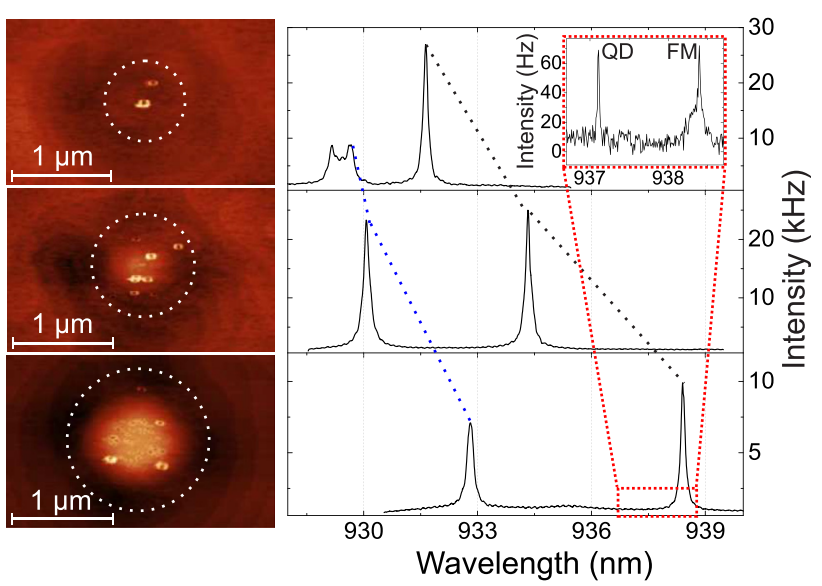

FIG. 2. Left: Atomic-force microscopy (AFM) images of a reference sample, demonstrating that the number of positioned QDs increases with the increasing aperture diameter, which is marked by a white dashed circle. Right: $\mu \mathrm{PL}$ spectra of micropillars with an identical aperture size (similar number of SCQDs) as shown on the left side and with a pillar diameter of $4.4 \mu \mathrm{m}$ taken at an excitation power of $0.22 \mathrm{~mW}$. Spectral shifts of the fundamental and the first higher order transverse modes for different aperture diameters are indicated by dashed lines. Inset: spectrum taken from a structure with an aperture diameter of $1400 \mathrm{~nm}$ at a lower excitation power of $12 \mu \mathrm{W}$ showing a narrow resolution limited QD line at $937.1 \mathrm{~nm}$ and emission of the fundamental pillar mode at $938.4 \mathrm{~nm}$ (the corresponding spectral region is marked with a red dashed line). 
aperture of $1400 \mathrm{~nm}$. Noteworthily, no QD-growth appears outside the aperture region, demonstrating a high selectivity of our growth technique. On the right side of Fig. 2, $\mu \mathrm{PL}$ spectra of micropillars with a diameter of $4.4 \mu \mathrm{m}$ and nominally same aperture diameters as on the left side are presented. We observe emission from the fundamental pillar mode on the low-energy side and from the first higher transverse mode on the high-energy side. Here, the mode assignment is based on the typical fingerprint of cavity emission with the fundamental mode having the lowest emission energy. ${ }^{13}$ The inset shows a low excitation power $\mu \mathrm{PL}$ spectrum of a SCQD with a resolution limited linewidth of $32 \mu \mathrm{eV}$ at $937.1 \mathrm{~nm}$ and fundamental pillar mode emission at $938.4 \mathrm{~nm}$. Together with the results obtained from similar SCQDs under strict resonant excitation published in our previous work, ${ }^{20}$ a high optical quality of the grown SCQDs is demonstrated. Interestingly, for the given pillar diameter, both the emission energy and the mode splitting depend on the aperture diameter which we attribute to its influence on the mode volume of the micropillar cavity. The oxideaperture's influence on the emission features is investigated experimentally for a family of micropillars with a constant diameter of $4.4 \mu \mathrm{m}$ and aperture diameters in the range of 500 to $1500 \mathrm{~nm}$. Additionally, numerical simulations are performed for aperture diameters in the range of 0 to $4400 \mathrm{~nm}$ by using the finite-element software package JCMsuite ${ }^{23}$ for solving second-order Maxwell's equations of the electric field. ${ }^{24}$ The results of these studies are presented in Fig. 3 where different parameters, such as the mode splitting $\Delta \lambda$, the quality factor $Q$, the mode volume $V_{\mathrm{m}}$, the Purcell factor $F_{\mathrm{P}}$,

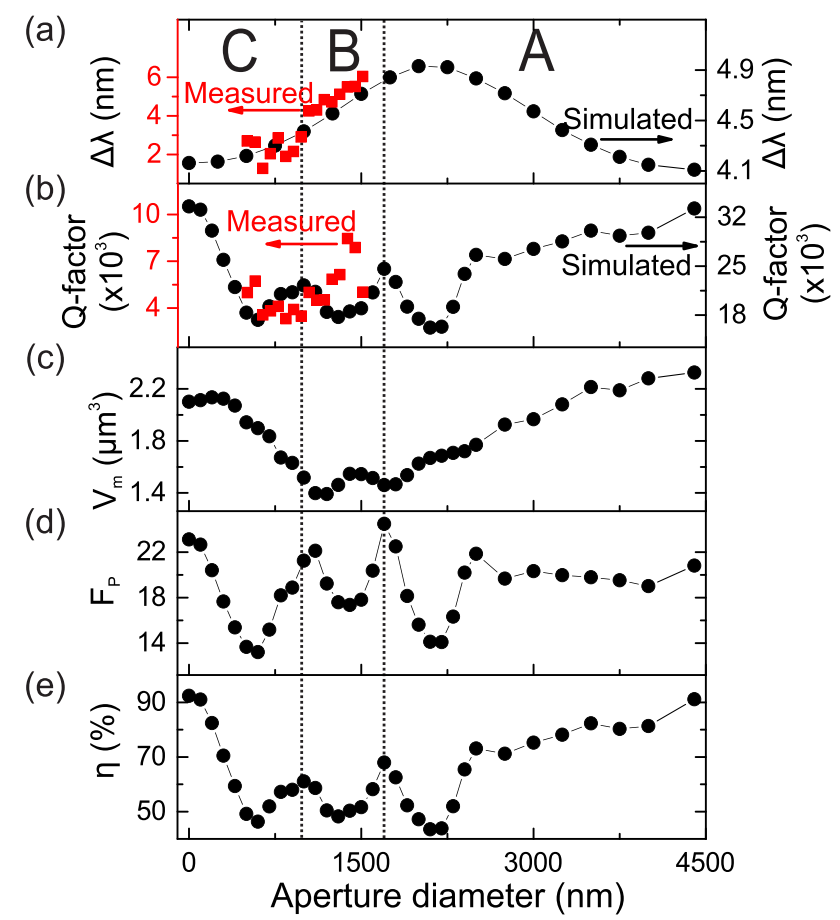

FIG. 3. Numerically simulated (black circles, labels on the right axis) and experimentally measured (red squares, left axis) mode splitting $\Delta \lambda$ (a) and $Q$-factor (b) as a function of the aperture diameter. Simulated mode volume $V_{\mathrm{m}}$ (c), Purcell factor $F_{\mathrm{P}}(\mathrm{d})$, and photon-extraction efficiency $\eta$ (e) as a function of the aperture diameter. All results correspond to the micropillars with a diameter of $4.4 \mu \mathrm{m}$. All plots are subdivided into regions A-C as described in the text. and the photon-extraction efficiency $\eta$, are depicted as a function of the aperture diameter. We subdivide the presented results into three characteristic diameter ranges A-C separated by dashed vertical lines (cf. Fig. 3). The simulation results of the mode behavior for the aperture diameters between 1700 and $4400 \mathrm{~nm}$ (region A) are in agreement with previous works ${ }^{25,26}$ and show an increase in the mode splitting $\Delta \lambda$ [Fig. 3(a)] and a reduction in the mode volume $V_{\mathrm{m}}$ [Fig. 3(c)] for a decreasing aperture diameter, which is explained by enhanced mode confinement. The latter leads to a blue-shift of the energy of the fundamental mode ${ }^{27}$ (not shown here). Due to light scattering induced by the refractive index contrast between the oxidized and non-oxidized materials, the $Q$-factor decreases with the decreasing aperture diameter [Fig. 3(b)]. ${ }^{28}$ In contrast, the Purcell factor $F_{\mathrm{P}}$ does not show a systematic dependence on the oxide-aperture diameter [Fig. 3(d)] by mutually compensating the influence of the decreasing $V_{\mathrm{m}}$ and $Q$.

For diameter regions $\mathrm{B}$ and $\mathrm{C}$, the theoretical findings are compared with the experimental data. In intermediate region B corresponding to aperture diameters between 800 and $1700 \mathrm{~nm}$, the mode confinement starts to decrease and $\Delta \lambda$ decreases accordingly. Here, the scattering-induced reduction of the experimental $Q$-factor is confirmed qualitatively by the simulation and results in a reduced photonextraction efficiency $\eta$ [cf. Fig. 3(e)], which is expressed as $\frac{Q_{\mathrm{MP}}}{Q_{0}} \frac{F_{\mathrm{P}}}{F_{\mathrm{P}}+1},{ }^{29}$ where $Q_{\mathrm{MP}}$ and $Q_{0}$ are the $Q$-factors of the micropillar and the planar structure, respectively. In region $\mathrm{C}$, the shrinking and possibly closing aperture lead to an increase in $V_{\mathrm{m}}$ as well as $Q$ and, hence, $\eta$. This observation is explained by a decoupling of the mode from the refractive index contrast associated with oxidized AlAs when the aperture diameter becomes smaller than the lateral mode extension. The oscillatory behavior of the $Q$-factor, $F_{\mathrm{P}}$, and $\eta$ for the aperture diameters $<2.5 \mu \mathrm{m}$ is attributed to a varying modematching of the mode in the cavity with propagative Bloch modes in the DBRs. ${ }^{24,30,31}$ The deviation of the measured $Q$ factors from the values predicted by the simulations is attributed partly to absorption losses, scattering losses at layer interfaces of the overgrown microcavity, and photon losses at the micropillar's sidewalls, which are not considered in the simulations.

In order to demonstrate pronounced single-QD cQED effects and to confirm the predicted enhancement of the spontaneous emission rate [cf. Fig. 3(d)], we performed temperature-tuning of a single-QD exciton (X) through the fundamental cavity mode (C) of a micropillar with a diameter of $4.4 \mu \mathrm{m}$ and an aperture diameter of $1.3 \mu \mathrm{m}$. Figure 4(a) presents the corresponding $\mu \mathrm{PL}$ spectra in the temperature range between 24 and $43 \mathrm{~K}$. At resonance at $35 \mathrm{~K}$, we observe pronounced enhancement of QD-X emission due to the Purcell effect. In order to determine the associated Purcell factor $F_{\mathrm{P}}$, we extracted the detuning dependent integrated intensity of $\mathrm{X}$ emission by Lorentzian lineshape fitting. The corresponding data are plotted in Fig. 4(b) as a function of the spectral detuning $\Delta$ between $\mathrm{X}$ and $\mathrm{C}$. By fitting this dependence with $I(\Delta)=F_{\mathrm{P}} /\left(1+F_{\mathrm{P}}+4 \Delta^{2} / \gamma_{\mathrm{C}}^{2}\right),{ }^{32}$ where $\gamma_{C}$ is the cavity linewidth, we determine $F_{\mathrm{P}}=4.3 \pm 0.3$. This value is lower in comparison to the simulated value of $F_{\mathrm{P}}=18$ for an ideal structure. Taking the experimental $Q$-factor into 


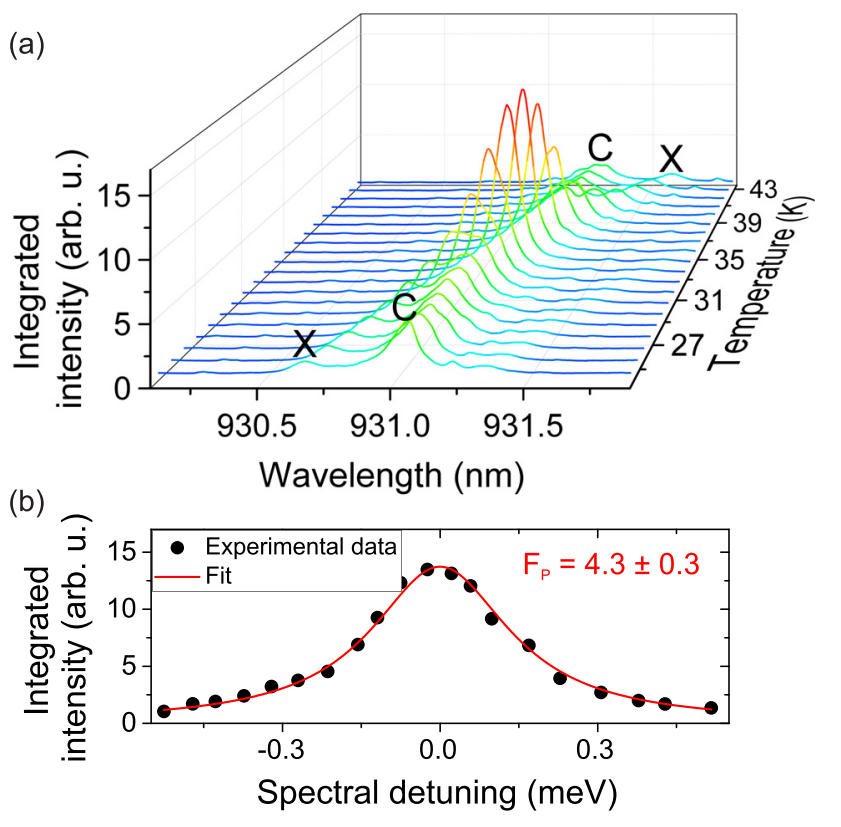

FIG. 4. (a) Color waterfall presentation of temperature dependent $\mu$ PL spectra showing the spectral tuning of a single-QD exciton $(\mathrm{X})$ through the resonance of the fundamental cavity mode (C) of a $4.4 \mu \mathrm{m}$ micropillar with an oxide-aperture diameter of $1300 \mathrm{~nm}$ and a $Q$-factor of 8000 . cQED enhanced light-matter interaction results in pronounced Purcell enhancement at the resonance temperature of $35 \mathrm{~K}$. (b) Integrated intensity of the QD emission as a function of the spectral detuning between $\mathrm{X}$ and $\mathrm{C}$. The fit according to the equation $I(\Delta)=F_{\mathrm{P}} /\left(1+F_{\mathrm{P}}+4 \Delta^{2} / \gamma_{\mathrm{C}}^{2}\right)$ (red line) yields $F_{\mathrm{P}}=4.3 \pm 0.3$.

account, the upper limit of the Purcell factor can be calculated via $F_{\mathrm{P}}=\left(3 Q_{\exp } / 4 \pi^{2} V_{\mathrm{M}}\right)\left(\lambda^{3} / n_{\mathrm{eff}}^{3}\right)^{1}$ which yields $F_{\mathrm{P}}^{\max }=11$. This value is still higher than the experimental $F_{P}$ which we attribute to a possible polarization mismatch between $\mathrm{X}$ and $\mathrm{C}$ and to a non-ideal spatial matching between the QD and the maximum of the fundamental micropillar mode. The latter has already been estimated in our previous work ${ }^{22}$ where the average displacement from the center of the micropillar was determined to be $640 \mathrm{~nm}$ for the aperture diameter from 1200 to $1600 \mathrm{~nm}$. We would like to point out that in the case of micropillar cavities, this effect has only a moderate impact in terms of cQED effects, resulting in a slightly reduced overlap between the vacuum electric field amplitudes at the antinode of the electromagnetic field and at the QD location which is still larger than $60 \%$. Moreover, considering that the displacement depends on the aperture diameter as the tensile strain maximum is located at the aperture boundaries, the mismatch can be further reduced.

Additionally, we verify the single-photon emission character of the QD-micropillar system by second-order autocorrelation measurements under pulsed non-resonant excitation (at $895 \mathrm{~nm}$ ) yielding $\mathrm{g}^{(2)}(\tau=0)=0.37 \pm 0.01$ (not shown), where the non-ideal multi-photon suppression is attributed to uncorrelated background emission of the cavity mode fed by off-resonant spectator QDs.

In conclusion, we presented an attractive fabrication platform for the realization of micropillar cavities with a controlled number of SCQDs in the active layer. The structures are fabricated by the buried-stressor growth concept and studied comprehensively in terms of the influence of the buried oxide-aperture on the optical properties of the micropillars. Both the $Q$-factor and the mode volume depend in a characteristic way on the diameter of the oxide-aperture. The latter leads to additional mode-confinement and scattering for a given micropillar diameter, thereby influencing the mode volume, the $Q$-factor, the associated Purcell-factor and, as a result the photon-extraction efficiency. The fabricated micropillars exhibit $Q$-factors of up to 12000 and a pronounced single-QD Purcell enhancement with $F_{\mathrm{P}}=4.3 \pm 0.3$. The demonstrated approach is well suited for the realization of cQED-enhanced SPSs and paves the way for the development of high- $\beta$ microlasers with a controlled number of SCQDs spatially aligned to the laser mode.

The research leading to these results received funding from the European Research Council under the European Union's Seventh Framework Program Grant Agreement No. 615613, from the Volkswagen Foundation via NeuroQNet, and from the German Research Foundation via CRC 787.

${ }^{1}$ J. M. Gérard, B. Sermage, B. Gayral, B. Legrand, E. Costard, and V. Thierry-Mieg, Phys. Rev. Lett. 81, 1110 (1998).

${ }^{2}$ S. Noda, M. Fujita, and T. Asano, Nat. Photonics 1, 449 (2007).

${ }^{3}$ P. Michler, A. Kiraz, C. Becher, W. V. Schoenfeld, P. M. Petroff, L. Zhang, E. Hu, and A. Imamoglu, Science 290, 2282 (2000).

${ }^{4}$ T. Heindel, C. Schneider, M. Lermer, S. H. Kwon, T. Braun, S. Reitzenstein, S. Höfling, M. Kamp, and A. Forchel, Appl. Phys. Lett. 96, 011107 (2010).

${ }^{5}$ O. Gazzano, S. Michaelis de Vasconcellos, C. Arnold, A. Nowak, E. Galopin, I. Sagnes, L. Lanco, A. Lemaître, and P. Senellart, Nat. Commun. 4, 1425 (2013).

${ }^{6}$ A. Schlehahn, A. Thoma, P. Munnelly, M. Kamp, S. Höfling, T. Heindel, C. Schneider, and S. Reitzenstein, APL Photonics 1, 011301 (2016).

${ }^{7}$ X. Ding, Y. He, Z.-C. Duan, N. Gregersen, M.-C. Chen, S. Unsleber, S. Maier, C. Schneider, M. Kamp, S. Höfling, C.-Y. Lu, and J.-W. Pan, Phys. Rev. Lett. 116, 020401 (2016).

${ }^{8}$ S. Unsleber, Y.-M. He, S. Gerhardt, S. Maier, C.-Y. Lu, J.-W. Pan, N. Gregersen, M. Kamp, C. Schneider, and S. Höfling, Opt. Express 24, 8539 (2016).

${ }^{9}$ S. Strauf, K. Hennessy, M. T. Rakher, Y.-S. Choi, A. Badolato, L. C. Andreani, E. L. Hu, P. M. Petroff, and D. Bouwmeester, Phys. Rev. Lett. 96, 127404 (2006).

${ }^{10}$ I. Prieto, J. M. Llorens, L. E. Muñoz-Camúñez, A. G. Taboada, J. CanetFerrer, J. M. Ripalda, C. Robles, G. Muñoz-Matutano, J. P. MartínezPastor, and P. A. Postigo, Optica 2, 66 (2015).

${ }^{11}$ S. Reitzenstein, T. Heindel, C. Kistner, A. Rahimi-Iman, C. Schneider, S. Höfling, and A. Forchel, Appl. Phys. Lett. 93, 061104 (2008).

${ }^{12}$ S. Reitzenstein, C. Böckler, A. Bazhenov, A. Gorbunov, A. Löffler, M. Kamp, V. D. Kulakovskii, and A. Forchel, Opt. Express 16, 4848 (2008).

${ }^{13}$ S. Reitzenstein and A. Forchel, J. Phys. D: Appl. Phys. 43, 033001 (2010).

${ }^{14}$ A. Dousse, L. Lanco, J. Suffczyński, E. Semenova, A. Miard, A. Lemaître, I. Sagnes, C. Roblin, J. Bloch, and P. Senellart, Phys. Rev. Lett. 101, 267404 (2008).

${ }^{15}$ C. Schneider, M. Strauß, T. Sünner, A. Huggenberger, D. Wiener, S. Reitzenstein, M. Kamp, S. Höfling, and A. Forchel, Appl. Phys. Lett. 92, 183101 (2008).

${ }^{16}$ A. Surrente, P. Gallo, M. Felici, B. Dwir, A. Rudra, and E. Kapon, Nanotechnology 20, 415205 (2009).

${ }^{17}$ F. Albert, S. Stobbe, C. Schneider, T. Heindel, S. Reitzenstein, S. Höfling, P. Lodahl, L. Worschech, and A. Forchel, Appl. Phys. Lett. 96, 151102 (2010).

${ }^{18}$ A. Huggenberger, S. Heckelmann, C. Schneider, S. Höfling, S. Reitzenstein, L. Worschech, M. Kamp, and A. Forchel, Appl. Phys. Lett. 98, 131104 (2011).

${ }^{19}$ A. Strittmatter, A. Holzbecher, A. Schliwa, J.-H. Schulze, D. Quandt, T. D. Germann, A. Dreismann, O. Hitzemann, E. Stock, I. A. Ostapenko, S. Rodt, W. Unrau, U. W. Pohl, A. Hoffmann, D. Bimberg, and V. Haisler, Phys. Status Solidi A 209, 2411 (2012).

${ }^{20}$ M. Strauß, A. Kaganskiy, R. Voigt, P. Schnauber, J.-H. Schulze, S. Rodt, A. Strittmatter, and S. Reitzenstein, Appl. Phys. Lett. 110, 111101 (2017).

${ }^{21}$ W. Unrau, D. Quandt, J.-H. Schulze, T. Heindel, T. D. Germann, O. Hitzemann, A. Strittmatter, S. Reitzenstein, U. W. Pohl, and D. Bimberg, Appl. Phys. Lett. 101, 211119 (2012). 
${ }^{22}$ A. Kaganskiy, S. Fischbach, A. Strittmatter, T. Heindel, S. Rodt, and S. Reitzenstein, Opt. Commun. 413, 162-166 (2018).

${ }^{23} \mathrm{JCM}$ suite software package version 3.2.1, JCMwave GmbH, 2016.

${ }^{24}$ M. Karl, B. Kettner, S. Burger, F. Schmidt, H. Kalt, and M. Hetterich, Opt. Express 17, 1144 (2009).

${ }^{25}$ M. P. Bakker, D. J. Suntrup, H. Snijders, T.-A. Truong, P. M. Petroff, D. Bouwmeester, and M. P. van Exter, Opt. Lett. 38, 3308 (2013).

${ }^{26}$ A. J. Bennett, D. J. P. Ellis, A. J. Shields, P. Atkinson, I. Farrer, and D. A. Ritchie, Appl. Phys. Lett. 90, 191911 (2007).

${ }^{27}$ D. J. P. Ellis, A. J. Bennett, S. J. Dewhurst, P. Atkinson, C. A. Nicoll, D. A. Ritchie, and A. J. Shields, J. Phys.: Condens. Matter 20, 454212 (2008).
${ }^{28}$ S. Weidenfeld, W.-M. Schulz, C. A. Kessler, M. Reischle, M. Eichfelder, M. Wiesner, M. Jetter, and P. Michler, Appl. Phys. Lett. 102, 011132 (2013).

${ }^{29}$ W. L. Barnes, G. Björk, J. M. Gérard, P. Jonsson, J. Wasey, P. T. Worthing, and V. Zwiller, Eur. Phys. J. D 18, 197 (2002).

${ }^{30}$ P. Lalanne, J. P. Hugonin, and J. M. Gérard, Appl. Phys. Lett. 84, 4726 (2004).

${ }^{31}$ S. Reitzenstein, N. Gregersen, C. Kistner, M. Strauss, C. Schneider, L. Pan, T. R. Nielsen, S. Höfling, J. Mørk, and A. Forchel, Appl. Phys. Lett. 94, 061108 (2009).

${ }^{32}$ M. Munsch, A. Mosset, A. Auffèves, S. Seidelin, J. P. Poizat, J.-M. Gérard, A. Lemaître, I. Sagnes, and P. Senellart, Phys. Rev. B 80, 115312 (2009). 